\title{
Tracing Control for a Tracked Vehicle Based on a Virtual Wheeled Mobile Robot
}

\author{
Masamitsu Kurisu, Kazutaka Takahashi \\ and Toyohiro Konishi \\ Dpt. of Mechanical Engineering \\ Tokyo Denki University \\ Kanda-Nishiki-cho 2-2, Chiyoda-ku \\ Tokyo, 101-8457, Japan \\ kurisu@ cck.dendai.ac.jp
}

\author{
Sigeru Sarata \\ Intelligent Systems Institute \\ Advanced Industrial Science and Technology(AIST) \\ Namiki 1-2-1, Tsukuba \\ Ibaraki, 305-8564, Japan \\ sarata-s@aist.go.jp
}

\begin{abstract}
Although a tracked vehicle enables stable movements on rough terrain, in a rotating motion no fixed kinematic model such as the model of wheeled mobile robot exists. Hence, it is difficult to realize a autonomous driving control of the tracked vehicle. In this paper, a new tracking control method for a tracked vehicle is described. The proposed method is constructed by using a virtual wheeled mobile robot.

A instantaneous motion of the vehicle with slip can be regarded as a motion of a mobile robot with independent driving wheels. From the kinematic constraints, the virtual mobile robot model is derived. The virtual desired trajectory for the virtual robot is obtained from the given desired trajectory for the original tracked vehicle under the assumption that the virtual model is fixed. A control rule is derived by applying a differential feedback control method for wheeled mobile robots, and guarantees that the virtual mobile robot follows the virtual desired trajectory. We also describe a on-line prediction method for the virtual robot so as to discard the assumption that the virtual model is fixed.
\end{abstract}

Keywords: Tracked vehicle, Tracking control, Wheeled mobile robot

\section{INTRODUCTION}

Tracked vehicle has a high road-hugging property compared with wheeled vehicles, because its crawlers make a field contact or points contact with ground. To the contrary, when the vehicle has a rotating motion, many regions of the crawler have to slip against the contact ground. Since the slip motion is different according to contact conditions and is unpredictable, there is no fixed kinematic model for the tracked vehicle such as the model of wheeled mobile robot. Hence, it is difficult to realize a autonomous driving control of the tracked vehicle.

This paper presents a new control method for a tracked vehicle to track a given trajectory. the proposed method is based on the control rule for a virtual wheeled mobile robot. The paper is organized as follows: In the next section the kinematic model of the vehicle with slip is discussed. The instantaneous motion of the vehicle with slippage can be regarded as a motion of a mobile robot with independent driving wheels. The virtual wheeled mobile robot is derived from the vehicle motion as the kinematic model. In the third section, the control method for the tracked vehicle with slip is proposed. Kinematic restrictions clarify that the vehicle with slippage can not no longer track the given trajectory strictly. The desired posture of the vehicle is slightly altered so that the position of the vehicle's origin can follow the trajectory. Then, the virtual desired trajectory for the virtual robot is derived from the altered trajectory under the assumption that the virtual model is fixed. Finally, a control rule for the tracked vehicle is derived by applying a differential feedback control method for wheeled mobile robots. the control method guarantees that the virtual mobile robot fol- lows the virtual desired trajectory. In the fourth section, the prediction method of the virtual robot is described so as to discard the assumption mentioned in previous section. The parameters concerning the virtual mobile robot are the position of its origin with respect to the original vehicle, and the tread length between two virtual wheels. These parameters will change if the property of ground or the contact condition between crawler and ground changes. However, when the motion of the vehicle shifts to clockwise rotation from counterclockwise rotation, or the reverse motion occurs, the parameters cause the discontinuity on representation. To avoid this, the parameters are projected on a unit sphere and converted to the polar-coordinate representation. The converted parameters have the continuity and linearity. The parameters are predicted by using a linear time series model. The control rule mentioned in the section 3 is applied on the predicted parameters. Final section makes conclusions and presents the future work.

\section{KINEMATIC MODEL}

First, differences of motion between a tracked vehicle and a mobile robot with independent driving wheels are discussed.

Figure 1 shows a typical model of the mobile robot. In the figure, $\Sigma_{R}\left(O_{R}-X_{R} Y_{R}\right)$ means the robot coordinate frame fixed to the robot with its origin at the intersection between the wheel axile and the center line of the robot. $Y_{R}$ axis of the frame is on the wheel axile. The tread width, which is the distance between the right and left wheels, is denoted by $T$. As known well, in constructing a kinematic model of the mobile robot, it is usual to make following assumptions:

A.1 Each wheel is in contact with the ground at a point. 


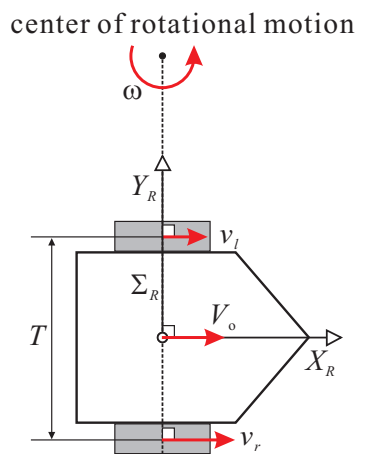

Figure 1: Typical model of wheeld mobile robot

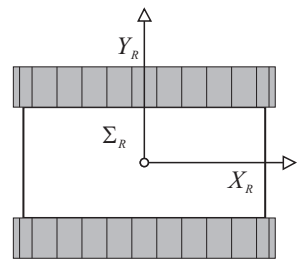

(a) Model of a tracked vehicle

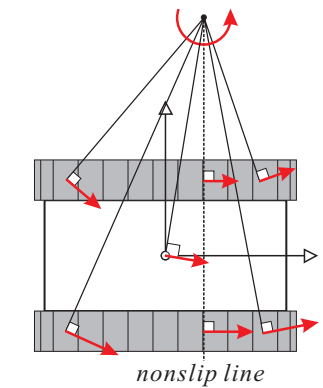

(a) Rotational motion (case 2)

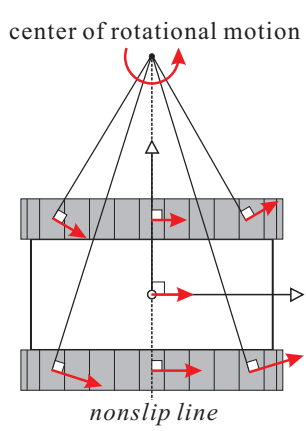

(b)Rotational motion (case 1)

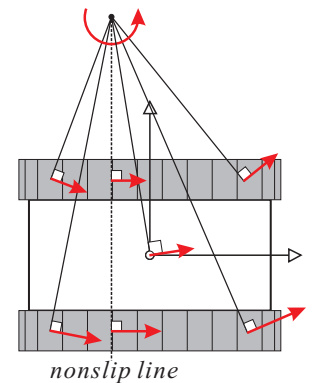

(b)Rotational motion (case 3)
Figure 2: Rotaitional motions of the tracked vehicle

A.2 No slip will occur at the point.

Under this assumptions, velocity on the perimeter of each wheel is perpendicular to the wheel axile. So, rotation centers of any motion of the robot are on the wheel axile. The velocity of the robot at the origin is also perpendicular to the wheel axile. After all, kinematic constraints obtained from the assumptions derive a following equation:

$$
\left[\begin{array}{l}
V_{o} \\
\omega
\end{array}\right]=\left[\begin{array}{cc}
\frac{1}{2} & \frac{1}{2} \\
\frac{1}{2 T} & -\frac{1}{2 T}
\end{array}\right]\left[\begin{array}{c}
v_{r} \\
v_{l}
\end{array}\right],
$$

where $V_{o}$ and $\omega$ are the translational and rotational velocity with respect to the robot respectively. Also, $v_{r}$ and $v_{l}$ are the velocities at the perimeter of right and left wheels respectively. The kinematic model of the wheeled mobile robot is described by the equation (1).

Figure 2(a) shows a model of a tracked vehicle. In the figure, $\Sigma_{R}$ is the robot coordinate frame fixed to the robot with its origin at the center of the vehicle. $X_{R}$ axis of the frame is on the center line of the vehicle. Now, we consider a rotational motion of the vehicle with the center of rotation on the $Y_{R}$ axis, as shown in Figure 2(b). In this case, the velocity at the points, which are the intersection between $Y_{R}$ axis and the crawlers and are on the perimeter of crawlers, are parallel to $X_{R}$ axis. However, it is easy to find from kinematic constraints that velocities of another point on the crawlers are not parallel to $X_{R}$ axis. This means that the crawlers surely have the points on which slips occur. Therefore, on any motion of the tracked vehicle, the assumption A.1 and A.2 are not realized. Here after, we call the line which includes a center of rotational motion and points of crawlers without slip "nonslip line". The location of nonslip line may change according to the contact conditions between the crawlers and ground, as shown in Figure 2(c),(d). Moreover, it is difficult to predict the location. This is the reason that the tracked vehicle is difficult to be controled autonomously.

Next, we derive a kinematic model of a tracked vehicle similar to the kinematic model of the mobile robot. Figure 3 shows a case that nonslip line is not on $Y_{R}$ axis. Let ${ }^{R} \boldsymbol{v}_{o}=\left[\begin{array}{ll}{ }^{R} v_{o x} & R_{v_{o y}}\end{array}\right]^{T}$ denote the translational velocity at the center of the vehicle, respectively. Describing the position of center of rotation as ${ }^{R} \boldsymbol{p}_{c}=\left[{ }^{R} x_{c}{ }^{R} y_{c}\right]^{T}$, following equations can be obtained:

$$
{ }^{R} x_{c}=-\frac{R_{v_{o y}}}{\omega},{ }^{R} y_{c}=\frac{R_{v_{o x}}}{\omega} .
$$

Note that upper subscript $R$ means the values are expressed with respect to $\Sigma_{R}$. From the kinematic constraint, we can find the point $\mathrm{O}$ ' which is on the nonslip line and has following propaties:

Describing the position of $\mathrm{O}^{\prime}$ as ${ }^{R} \boldsymbol{p}_{o^{\prime}}=\left[\begin{array}{lll}{ }^{R} x_{o^{\prime}} & { }^{R} y_{o^{\prime}}\end{array}\right]^{T}$, the velocity at $\mathrm{O}^{\prime}$ as ${ }^{R} \boldsymbol{v}_{o^{\prime}}=\left[V_{o^{\prime}} 0\right]^{T}$, following constraints are fulfilled:

$$
\begin{gathered}
{ }^{R} x_{o^{\prime}}={ }^{R} x_{c}, \\
{ }^{R} y_{o^{\prime}} \omega V_{o^{\prime}}=\left({ }^{R} y_{o^{\prime}}-{ }^{R} y_{c}\right) \omega,
\end{gathered}
$$

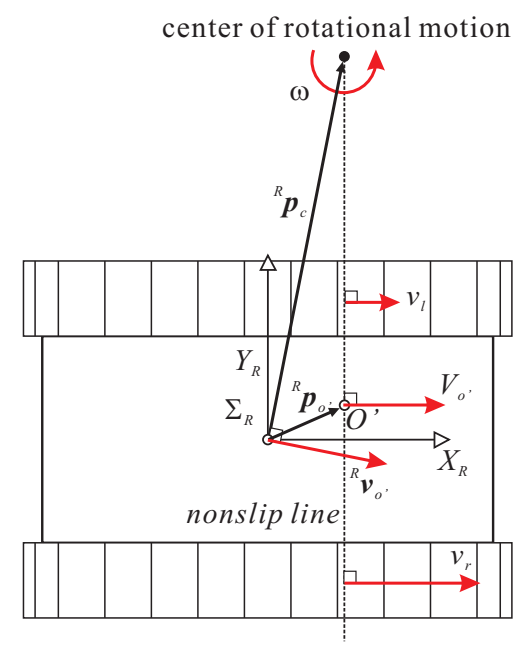

Figure 3: Kinematic constraints of the tracked vehicle 


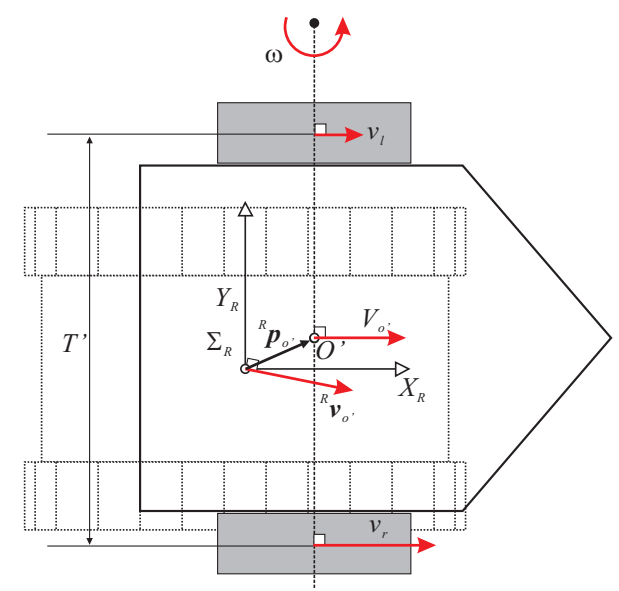

Figure 4: Virtual mobile robot

$$
\left[\begin{array}{c}
V_{o^{\prime}} \\
\omega
\end{array}\right]=\left[\begin{array}{cc}
\frac{1}{2} & \frac{1}{2} \\
\frac{1}{2 T^{\prime}} & -\frac{1}{2 T^{\prime}}
\end{array}\right]\left[\begin{array}{l}
v_{r} \\
v_{l}
\end{array}\right]
$$

Supposing that the velocity $\left({ }^{R} v_{o x}, R_{v_{o y}}, \omega\right)$ and $\left(v_{r}, v_{l}\right)$ can be measured, from the equation (3) (5), $R_{\boldsymbol{p}_{o^{\prime}}}$ and $T^{\prime}$ are given by

$$
\left\{\begin{array}{l}
R_{x_{o^{\prime}}}=-\frac{R_{v_{o y}}}{\omega} \\
R_{y_{o^{\prime}}}=\frac{v_{r}+v_{l}-2^{R} v_{o x}}{2 \omega} \\
T^{\prime}=\frac{v_{r}-v_{l}}{\omega}
\end{array}\right.
$$

Also, following relation is obtained

$$
V_{o^{\prime}}={ }^{R} y_{o^{\prime}} \omega+\sqrt{\left|R_{\boldsymbol{v}_{o}}\right|^{2}-\left({ }^{R} x_{o^{\prime}} \omega\right)^{2}}
$$

Conversely, at this time the motion of the tracked vehicle can be regarded as the motion of the virtual wheeled mobile robot whose center is on the point $\mathrm{O}^{\prime}$ and whose tread is $T^{\prime}$, as shown in Figure 4. The kinematic model of a tracked vehicle is described by the equation (5) and (6). This model is the similar one derived in the reference [1].

Our tracking control method is based on the virtual mobile robot. The parameter set of the virtual robot $\left({ }^{R} x_{o^{\prime}}, R_{y_{o^{\prime}}}, T^{\prime}\right)$ is varied according to the contact state between the crawlers and the ground. An adaptive prediction of the parameters is necessary in order to realize the tracking control. The prediction method will be described in the section 4.

\section{TRACKING CONTROL FOR TRACKED VEHI- CLE}

\section{1 Desired Trajectory}

As described in section 2, the motion of a tracked vehicle with slip can be regarded as the motion of the virtual mobile robot with two independent driving wheels. Since some tracking control methods for wheeled mobile robots have been proposed in the research fealds, the vehicle with slip may be able to control via a certain proposed control method by using the virtual mobile robot model. However, as one

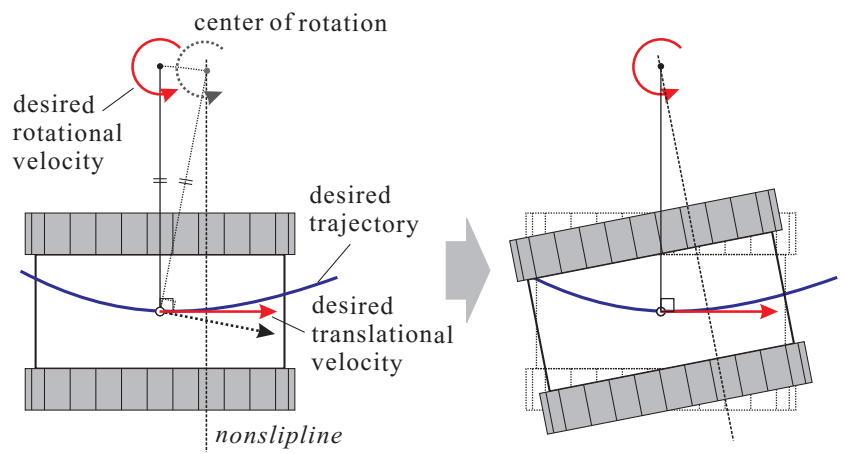

Figure 5: Alteration of desired posture

can see easily from kinematic restrictions, in a rotational motion with slip it is impossible to realize the velocity at the center of the vehicle to be the same as the desired velocity, because the nonslip line is shifted (see Figure 5). This means the vehicle with slip can not no longer track the given trajectory strictly. Therefore, it is necessary to alter the desired posture of the vehicle slightly so that the center of the vehicle can follow the trajectory. Here, the altered trajectory will be described.

An univarsal coordinate frame $\Sigma_{U}\left(O_{U}-X_{U} Y_{U}\right)$ is attached to the work ground. We consider that the desired trajectory for a tracked vehicle is given by the set of position, orientation, translational velocity and rotational one about the center of vehicle, which is denoted by $\left({ }^{U} x_{o d}(t),{ }^{U} y_{o d}(t), \theta_{o d}(t), V_{o d}(t), \omega_{o d}(t)\right)$ (see Figure 6). Position vector is defined by ${ }^{U} \boldsymbol{p}_{\text {od }}(t)=\left[{ }^{U} x_{o d}(t){ }^{U} y_{o d}(t)\right]^{T}$. We assum that $V_{o d}(t)>0(t>0)$. Also, following relations

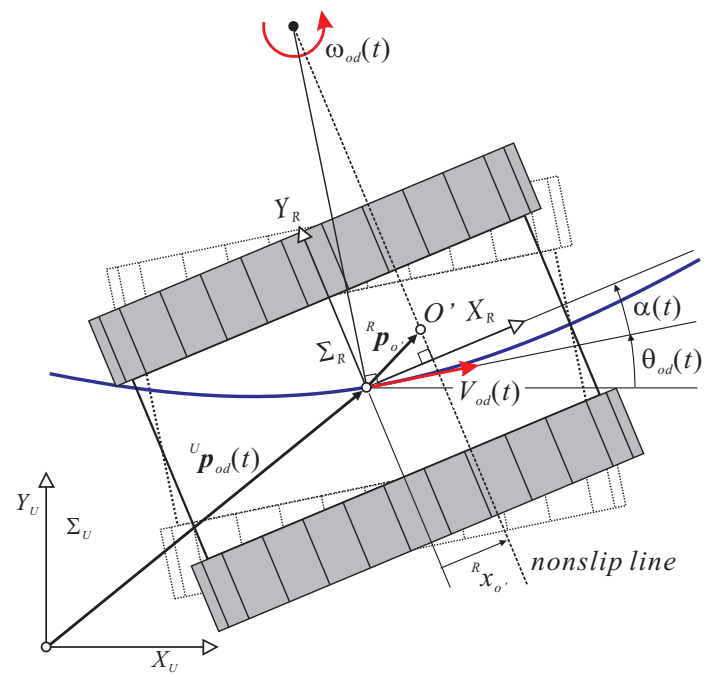

Figure 6: Desired trajectory and configuration of tracked vehicle 
assumed to be saticfied:

$$
\begin{aligned}
{ }^{U} x_{o d}(t) & =\int_{0}^{t} V_{o d}(\tau) \cos \theta_{o d}(\tau) d \tau+{ }^{U} x_{o d}(0) \\
U_{y_{o d}}(t) & =\int_{0}^{t} V_{o d}(\tau) \sin \theta_{o d}(\tau) d \tau+{ }^{U} y_{o d}(0) \\
\theta_{o d}(t) & =\int_{0}^{t} \omega_{o d}(\tau) d \tau+\theta_{o d}(0)
\end{aligned}
$$

Now, we assume that the virtual mobile robot model is fixed to $\left({ }^{R} x_{o^{\prime}},{ }^{R} y_{o^{\prime}}, T^{\prime}\right)$. Describing the angular displacement of orientation at ${ }^{U} \boldsymbol{p}_{o d}(t)$, which should be altered, by $\alpha(t), \alpha(t)$ is given by

$$
\alpha(t)=\sin ^{-1}\left(\frac{R_{x_{o^{\prime}} \omega_{o d}(t)}}{V_{o d}(t)}\right) .
$$

After all, the altered trajectory is given by $\left({ }^{U} x_{o d}(t),{ }^{U} y_{o d}(t)\right.$, $\left.\theta_{\text {od }}(t)+\alpha(t), V_{o d}(t), \omega_{o d}(t)\right)$.

\subsection{Control Algorithm}

In order to control the tracked vehicle with slip, eq.vrelation we apply a differential feedback control method for wheeled mobile robots proposed by Kanayama[2] to the virtual mobile robot. Since the center of the virtual robot, about which the control rule shoul be adopted, is different from the center of the original vehicle, the desired trajectory for the virtual robot is needed.

First, we derive the virtual desired trajectory for the virtual robot from the altered trajectory. In addition, in the following, the notation about time will be omitted. The virtual

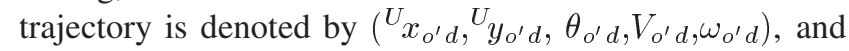
the position of the vertual robot's center with respect to $\Sigma_{U}$ is denoted by ${ }^{U} \boldsymbol{p}_{o^{\prime} d}$. In addition, in the following, the notation about time will be omitted. Since the displacement of the vertual center from the origin of $\Sigma_{R}$ is $R_{\boldsymbol{p}_{o^{\prime}}}$, the desired potision of the vertual center is given by

$$
{ }^{U} \boldsymbol{p}_{o^{\prime} d}={ }^{U} \boldsymbol{p}_{o d}+\boldsymbol{R}\left(\theta_{o d}+\alpha\right)^{R} \boldsymbol{p}_{o^{\prime}},
$$

where $\boldsymbol{R}(\theta)\left(\in \mathcal{R}^{2 \times 2}\right)$ is the rotation matrix described as follows

$$
\boldsymbol{R}(\theta)=\left[\begin{array}{cc}
\cos \theta & -\sin \theta \\
\sin \theta & \cos \theta
\end{array}\right] .
$$

Since $V_{o d}$ and $V_{o^{\prime} d}$ correspond to $\left|{ }^{R} \boldsymbol{v}_{o^{\prime}}\right|$ and $V_{o}$ in equation (7) respectively, the virtual trajectory is given by

$$
\left\{\begin{array}{l}
{ }_{x_{o^{\prime} d}}={ }^{U} x_{o d}+{ }^{R} x_{o^{\prime}} \cos \left(\theta_{o d}+\alpha\right)-{ }^{R} y_{o^{\prime}} \sin \left(\theta_{o d}+\alpha\right) \\
{ }_{y_{o^{\prime} d}}={ }^{U} y_{o d}+{ }^{R} x_{o^{\prime}} \sin \left(\theta_{o d}+\alpha\right)+{ }^{R} y_{o^{\prime}} \cos \left(\theta_{o d}+\alpha\right) \\
\theta_{o^{\prime} d}=\theta_{o d}+\alpha \\
V_{o^{\prime} d}={ }^{R} y_{o^{\prime}} \omega_{o d}+\sqrt{V_{o d}^{2}-\left({ }^{R} x_{o^{\prime}} \omega_{o d}\right)^{2}} \\
\omega_{o^{\prime} d}=\omega_{o d}
\end{array}\right.
$$

Next, we apply the differential feedback control rule for wheeled mobile robots proposed by Kanayama[2]. This control rule determines the translational and rotational velocities of the mobile robot according to the position and

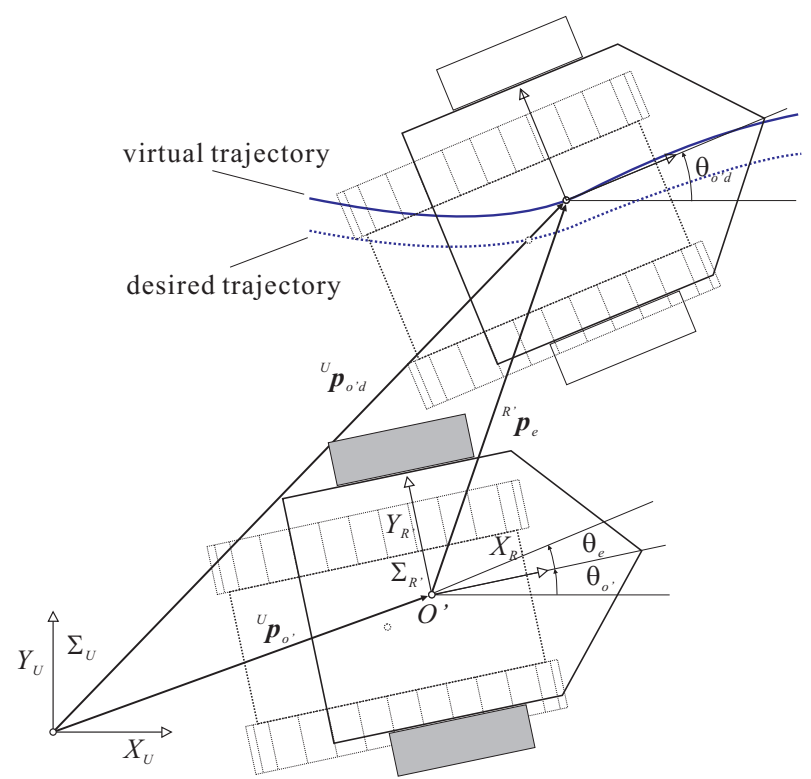

Figure 7: Position error of virtual center with respect to $\Sigma_{R^{\prime}}$

orientation errors with respect to the desired trajectory expressed in the coordinate frame fixed on the robot, and guarantees that the actual trajectory of the robot converges uniformly asymptotically to the desired trajectory.

Let $\Sigma_{R^{\prime}}$ denote the coordinate frame with its origin at the virtual center and its $X_{R^{\prime}}$ axis parallel to $X_{R}$ (see Figure 7). Then, we consider the position error of the virtual center expressed in $\Sigma_{R^{\prime}}$ with respect to the virtual trajectory. The error is denoted as ${ }^{R^{\prime}} \boldsymbol{p}_{e}=\left[{ }^{\prime} x_{e},{ }^{R} y_{e}\right]^{T}$. Denoting the actual position of the virtual center expressed in $\Sigma_{U}$ by ${ }^{U} \boldsymbol{p}_{o^{\prime}}$, the error ${ }^{\prime} p_{e}$ is given by

$$
{ }^{R^{\prime}} \boldsymbol{p}_{e}=\boldsymbol{R}\left(\theta_{o^{\prime} d}\right)\left({ }^{U} \boldsymbol{p}_{o^{\prime} d}-{ }^{U} \boldsymbol{p}_{o^{\prime}}\right),
$$

where ${ }^{U} \boldsymbol{p}_{o^{\prime}}$ is given by

$$
{ }^{U} \boldsymbol{p}_{o^{\prime}}={ }^{U} \boldsymbol{p}_{o}+\boldsymbol{R}\left(\theta_{o}+\alpha\right){ }^{R} \boldsymbol{p}_{o^{\prime}} .
$$

Denoting the actual orientation of the robot by $\theta_{o^{\prime}}$, the orientation error by $\theta_{e}=\theta_{o^{\prime} d}-\theta_{o^{\prime}}$, and adopting the differential feedback rule described by

$$
\left[\begin{array}{c}
V_{o^{\prime} \text { ref }} \\
\omega_{o^{\prime} \text { ref }}
\end{array}\right]=\left[\begin{array}{c}
V_{o^{\prime} d} \cos \theta_{e}+K_{x} R^{\prime} x_{e} \\
\omega_{o^{\prime} d}+V_{o^{\prime} d}\left(K_{y} R^{\prime} y_{e}+K_{\theta} \sin \theta_{e}\right)
\end{array}\right],
$$

it is guaranteed that $\boldsymbol{e}_{o^{\prime}}=\left[{ }^{R^{\prime}} \boldsymbol{p}_{e}^{T}, \theta_{e}\right]^{T}$ converges uniformly asymptotically to $\mathbf{0}$ as far as $V_{o^{\prime} d}>0$. In equation (17), $K_{x}, K_{y}$, and $K_{\theta}$ are feedback parameters, the crawlersand $V_{o^{\prime} r e f}, \omega_{o^{\prime} \text { ref }}$ are reference velocities of the virtual robot.

When the desired trajectory is a linear motion with constant velocity given by ${ }^{U} \boldsymbol{p}_{o^{\prime} d}(t)=[V t, 0]^{T}, \theta_{o^{\prime} d}(t)=0$, and initial condition $\theta_{e}(0)=0,1 / K_{x}$ corresponds to the time constant of the exponential decay concerning the initial position error ${ }^{\prime} x_{e}(0)$. Also, $K_{\theta} /\left(2 \sqrt{K_{y}}\right)$ and $V \sqrt{K_{y}}$ correspond to the damping coefficient and natural circular frequency of ${ }^{U} y_{o^{\prime}}(t)$ respectively. (For more information, see the reference 3 .) 
Finally, substituting equation (17) to equation (5), the reference verocities for the crawlers of actual tracked vehicle are derived as follows:

$$
\left[\begin{array}{l}
v_{\text {rref }} \\
v_{\text {lref }}
\end{array}\right]=\left[\begin{array}{cc}
\frac{1}{2} & \frac{1}{2} \\
\frac{1}{2 T^{\prime}} & -\frac{1}{2 T^{\prime}}
\end{array}\right]^{-1}\left[\begin{array}{l}
V_{o^{\prime} \text { ref }} \\
\omega_{o^{\prime} r e f}
\end{array}\right]
$$

Consequently, if we set the reference verocities $v_{r}$ ref and $v_{l \text { ref }}$ to the crawler as control inputs, we can expect to make the tracked vehicle follow the desired trajectory.

\section{PREDICTION OF VIRTUAL CENTER}

In the previous section, The tracking control method was derived under the assumption the virtual robot is fixed. That is to say the kinematic model of the tracked vehicle expressed the parameters $\left({ }^{R} x_{o^{\prime}},{ }^{R} y_{o^{\prime}}, T^{\prime}\right)$ is never altered. However, these parameters will change if the property of ground or the contact condition between crawlers and ground changes. A certain estimation about the parameters is needed to control the vehicle in practical use. In this section, the prediction method of the parameters is described so as to discard the assumption.

When the motion of the vehicle shifts to a clockwise rotation from a counterclockwise rotation, or the reverse motion occurs, the location of the virtual center moves to a negative infinite point from a positive infinite point, or to a positive from a negative. Namely, the parameters cause the discontinuity on representation. To avoid this, the parameters are projected on a unit sphere and converted to the polarcoordinate representation.

Consider two planes which contact with a unit sphere at the origin of the vehicle (see Figure 8). The upper plane corresponds to the counterclockwise rotation, and the bottom plane corresponds to the clockwise rotation. A coordinate frame $\Sigma_{S}\left(O_{S}-X_{S} Y_{S} Z_{S}\right)$ is attached to the sphere with its origin at the center of the sphere. The $X_{S}$ axis is alined to $X_{R}$ of the upper plane. The $Z_{S}$ axis is upward along a vertical line. Let $P_{s}$ denote the intersection of the spherical surface and the line which connects the virtual center $\left({ }^{R} x_{o^{\prime}},{ }^{R} y_{o^{\prime}}\right)$ to the origin of $\Sigma_{S}$. The coordinates

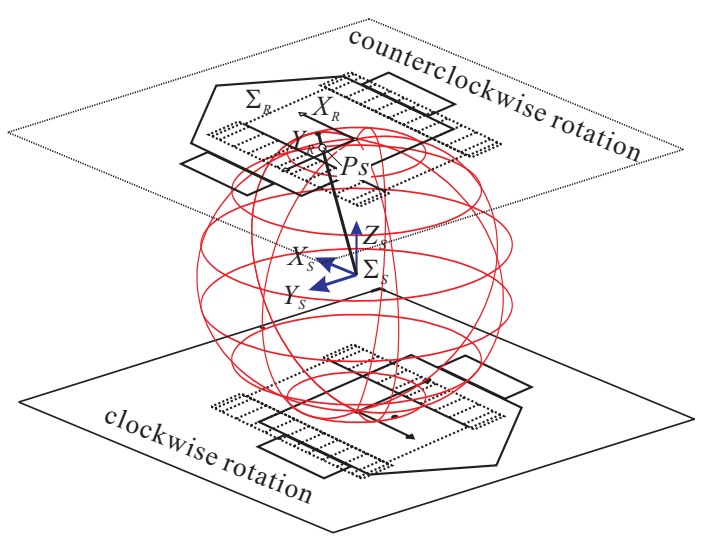

Figure 8: Projection to a unit sphere of $P_{s}\left({ }^{S} x_{s},{ }^{S} y_{s},{ }^{S} z_{s}\right)$ are given as follows:

$$
\left\{\begin{array}{l}
S_{x_{s}}=\frac{R_{v_{o y}}}{l}, \\
S_{y_{s}}=\frac{v_{r}+v_{l}-2^{R} v_{o x}}{l}, \\
S_{z_{s}}=\frac{\omega}{l}, \\
l=\sqrt{\left(v_{r}+v_{l}-2^{R} v_{o x}\right)^{2}+{ }^{R} v_{o y}^{2}+\omega^{2}} .
\end{array}\right.
$$

Also, the virtual center is represented by the coordinates of $P_{s}$

$$
R_{x_{o^{\prime}}}=\frac{{ }^{S} x_{s}}{S_{z_{s}}},{ }^{R} y_{o^{\prime}}=\frac{S_{y_{s}}}{S_{z_{s}}} .
$$

Then, $P_{s}$ is converted to the polar-coordinate representation $(\beta, \gamma) . \beta(-\pi \leq \beta \leq \pi)$ represents the longitude, and $\gamma(-\pi \leq \gamma \leq \pi)$ the latitude.

The converted parameters have continuity and linearity. These parameters are predicted by using a linear time series model, that is AR model. The virtual tread width $T^{\prime}$ is predicted by using AR model. The control rule will be applied on the predicted parameters.

\section{CONCLUSION}

This paper presented a new tracking control method for a tracked vehicle. The proposed method is constructed by using a virtual wheeled mobile robot.

A rotational motion of the vehicle with slip can be regarded as a motion of a mobile robot with independent driving wheels. The virtual wheeled mobile robot model was derived based on kinematics. The virtual trajectory for the model is obtained from the given desired trajectory for the original tracked vehicle. The control rule was derived by applying a differential feedback control method for wheeled mobile robots under the assumption that the virtual model is fixed. The control rule guarantees that the virtual robot follows the virtual trajectory. We also describe a prediction method for the virtual robot so as to discard the assumption that the virtual model is fixed.

As a future work, we will verify the effectiveness of the proposed method via experiments using a miniature tracked vehicle robot.

\section{REFERENCES}

[1] J.L. Martinez, A. Mandow, J. Morales, A. GarciaCwrezo and S. Pedraza: "Kinematic Modeling of Tracked Vehicles by Experimental Identification," in proceedings of IEEE/RSJ International Conference on Intelligent Robots and Systems IROS2004, pp.14871492, 2004.

[2] Y. Kanayama, Y. Kimura, F. Miyazaki and T. Noguchi, "A Stable Tracking Control Method for a Non-Holonomic Mobile Robot," in proceedings of IEEE/RSJ International Workshop on Intelligent Robots and Systems IROS'91, pp.1236-1241, 1991. 